\title{
Intraoperative real-time identification of abnormal vessels within the bright field by superselective arterial injection of saline and its slow-motion recording using a high frame rate digital camera during surgical treatment of spinal arteriovenous shunts: technical note
}

\author{
Shuji Hamauchi, MD, PhD, ${ }^{1}$ Toshiya Osanai, MD, PhD, ${ }^{1}$ Toshitaka Seki, MD, PhD, ${ }^{1}$ \\ Masahito Kawabori, MD, PhD, ${ }^{1}$ Michinari Okamoto, MD, ${ }^{1}$ Kazutoshi Hida, MD, PhD, ${ }^{2}$ and \\ Kiyohiro Houkin, MD, PhD' \\ 1Department of Neurosurgery, Hokkaido University Graduate School of Medicine; and 2Department of Neurosurgery, Sapporo \\ Azabu Neurosurgical Hospital, Sapporo, Hokkaido, Japan
}

The usefulness of superselective arterial injection of indocyanine green (SAI-ICG) during the surgical treatment of a spinal arteriovenous shunts (AVSs) has occasionally been reported. However, SAI-ICG lacks the temporal resolution for recording rapid blood flow. The authors found that SAI of saline (SAIS) via a microcatheter renders the vessels transparent because of the replacement of intravascular blood with saline. By combining SAIS with SAI-ICG, they were able to identify abnormal vessels in the bright field during the surgical treatment of spinal AVS. Furthermore, they performed slow-motion video recording of blood flow in abnormal vessels by mounting a high frame rate (HFR) digital camera on the microscope eyepiece during SAIS. In this paper, the authors present two cases of patients with spinal AVSs. The first patient had a cervical dural arteriovenous fistula, and the second had a thoracic spinal arteriovenous malformation. SAIS delineated abnormal vessels within the bright field in real time in both cases. HFR video was recorded in the second case during SAIS. The slow-motion video showed a smooth and seamless motion of the blood flow in the feeding artery, which facilitated an understanding of the blood flow direction.

https://thejns.org/doi/abs/10.3171/2018.3.SPINE1854

KEYWORDS spinal arteriovenous shunt; intraoperative angiography; slow-motion video; high frame rate; surgical technique; vascular disorders

$\mathrm{T}$ HE surgical treatment of spinal arteriovenous shunts (AVSs) is sometimes challenging because of their complex angioarchitecture. Correct intraoperative identification of the feeding artery and the draining vein is important in order to disconnect an arteriovenous fistula (AVF) or excise an arteriovenous malformation $(\mathrm{AVM})$ and preserve the normal spinal cord circulation. The superselective arterial injection of indocyanine green (SAI-ICG) is effective for the intraoperative identification of feeding arteries and draining veins. ${ }^{2,3}$ The frame rate of an ordinary recording system on an operating microscope lacks the temporal resolution for observing the rapid movement of blood flow. In this paper, we describe a method for the real-time identification of abnormal vessels within the bright field using SAI of saline (SAIS) through a microcatheter. Furthermore, we document the method of the slow-motion videorecording of the bloodstream in abnormal vessels using a high frame rate (HFR) digital camera for the detailed observation of the blood flow.

\section{Methods}

We performed the following procedure for the surgical treatment of 2 patients-one with a cervical dural AVF (dAVF) and one with a thoracic intramedullary AVM.

\section{Surgical Technique}

After the induction of general anesthesia, the inguinal

ABBREVIATIONS ASA = anterior spinal artery; $A V F=$ arteriovenous fistula; $A V M=$ arteriovenous malformation; $A V S=$ arteriovenous shunt; $d A V F=$ dural $A V F ; D S A=$ digital subtraction angiography; fps = frames per second; HFR = high frame rate; ICG = indocyanine green; NIR = near infrared; OPLL = ossification of the posterior longitudinal ligament; SAI = superselective arterial injection; SAI-ICG = SAI of ICG; SAIS = SAI of saline.

SUBMITTED January 12, 2018. ACCEPTED March 29, 2018.

INCLUDE WHEN CITING Published online August 10, 2018; DOI: 10.3171/2018.3.SPINE1854. 
region was sterilized and a 5-Fr metallic kink-resistant sheath (Super Allow-Flex, Teleflex Medical) was introduced into the left femoral artery. The sheath was fixed to the skin and draped to maintain sterile conditions. The patient was then turned to the prone position. The surgery level of the spinal cord was identified using a C-arm fluoroscope. Laminectomy and dural opening were performed, and the vascular lesion was visualized. After intraoperative fluorescence angiography, SAI-ICG and SAIS were performed to identify the feeding artery and the draining vein. Subsequently, the obliteration of the arterialized vein or excision of the nidus was performed. Motor evoked potentials were monitored during the surgery.

\section{Intraoperative Fluorescence Angiography, ICG Angiography, and Saline Injection}

A 4-Fr catheter was inserted into the 5-Fr metallic kink-resistant sheath and cannulated into the origin of the segmental artery from which the feeding artery originated. We used a 1.6-2.1-Fr microcatheter to prevent "catheter kickback" as mentioned in a previous report. ${ }^{2}$ For the superselective fluorescent angiography of the segmental artery, approximately $2 \mathrm{~mL}$ of a contrast medium was injected into the microcatheter. During SAI-ICG, the observation mode of the operating microscope (Zeiss, OPMI PENTERO 900) was changed from the bright field mode to the NIR (near-infrared) mode. Diluted (1:400-1:1200) ICG was injected through the microcatheter, and fluorescence signals of the vessels were observed in the display. SAI-ICG video was recorded in 29.97 interlaced frames per second (fps). For SAIS, approximately $2 \mathrm{ml}$ of heparinized saline solution was injected into the microcatheter via a $2.5-\mathrm{ml}$ syringe. The change in color of vessels induced by the injected saline is observed within the visual field of the microscope in SAIS.

\section{Video Recording With Digital Camera and High Frame Rate Recording}

During SAIS, the flow of injected saline was filmed at 960 fps by mounting a digital camera (Sony, RX100 V) on the microscope eyepiece (Fig. 1). To avoid missing the vessels turning transparent, the "endo-trigger mode" was used. In this mode, the camera captures 4 seconds of the video before the video button is pressed. The video recorded in the SD card was immediately viewed on a personal computer.

\section{Results}

We performed SAIS during surgical treatment of 2 patients with SASs - one with a cervical dAVF and one with a thoracic intramedullary AVM. Abnormal vessels were successfully identified with SAIS, and the arteriovenous shunt lesions were completely eradicated. No complication occurred in either case.

\section{Case 1}

This 78-year-old man presented with posterior cervical pain. Neurological examination showed no deficits. MRI of the cervical spine showed spinal canal stenosis from C2 -3 to C6-7 and a serpentine and dilated perimedullary

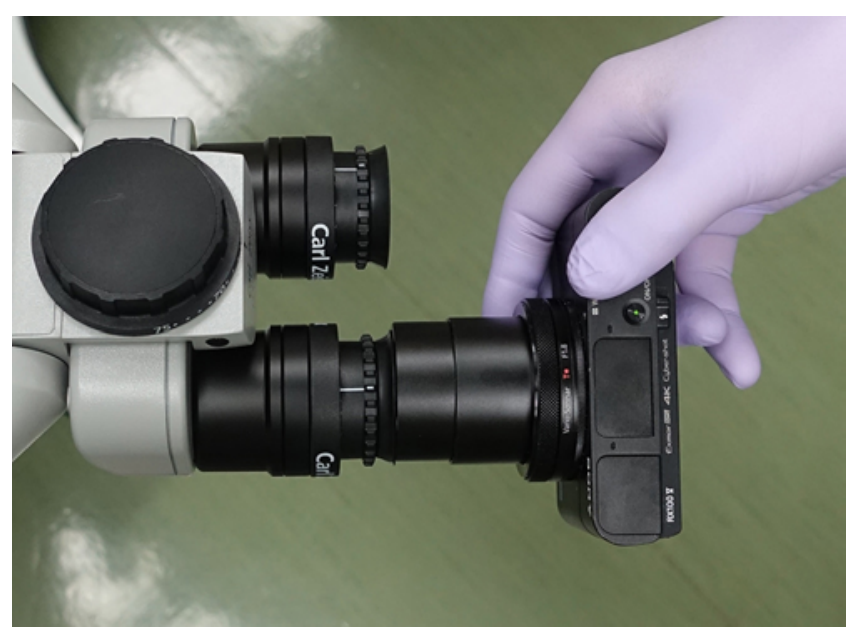

FIG. 1. For HFR recording, the digital camera (Sony RX100 V) is mounted onto the operating microscope eyepiece. Figure is available in color online only.

venous plexus in the dorsal spinal cord (Fig. 2A). Cervical CT showed segmental ossification of the posterior longitudinal ligament (OPLL) at the $\mathrm{C} 3$ and $\mathrm{C} 4$ vertebral levels (Fig. 2B). Dural or perimedullary AVF was suspected on the basis of CT angiography (Fig. 2B). Digital subtraction angiography (DSA) confirmed the diagnosis of dural AVF (dAVF) fed by the left C6 segmental artery (Fig. 2C).

Surgical treatment for spinal canal stenosis and dAVF was performed. After laminoplasty from C3 to C6, the dura mater was opened at the C6 spinal root level. A dilated vein running along the $\mathrm{C} 6$ spinal root was observed (Fig. 3A). SAIS from the left C6 segmental artery rendered the draining vein transparent in the bright field (Fig. 3B and Video 1), whereas SAI-ICG from the left C6 segmental artery showed fluorescence intensity in the dilated vein (Fig. 3C).

VIDEO 1. Superselective arterial injection of saline (SAIS) from the left $\mathrm{C} 6$ segmental artery changes the color of the draining vein, making it transparent in the bright field. Copyright Department of Neurosurgery, Hokkaido University Graduate School of Medicine.

Published with permission. Click here to view.

The draining vein was disconnected at the point where it emerged from the dura mater. Postoperatively the patient had no neurological deficit and his posterior cervical pain disappeared.

\section{Case 2}

This 29-year-old man suddenly developed paraplegia and was taken to another hospital where he was diagnosed with a thoracic intramedullary AVM and was referred to our hospital for further care. Neurological examination showed complete paraplegia, hyperalgesia below T6, and bladder and rectal disturbances. MRI showed a serpentine and dilated perimedullary venous plexus on the dorsal surface of the spinal cord and hemorrhagic change in the spinal cord at the T7-8 levels (Fig. 4A). CT angiography revealed an intramedullary AVM (Fig. 4B). DSA showed that the AVM was fed by a branch of the anterior spinal artery (ASA) originating from the left T6 segmental artery (Fig. 4C) and a posterior spinal artery (PSA) originating 


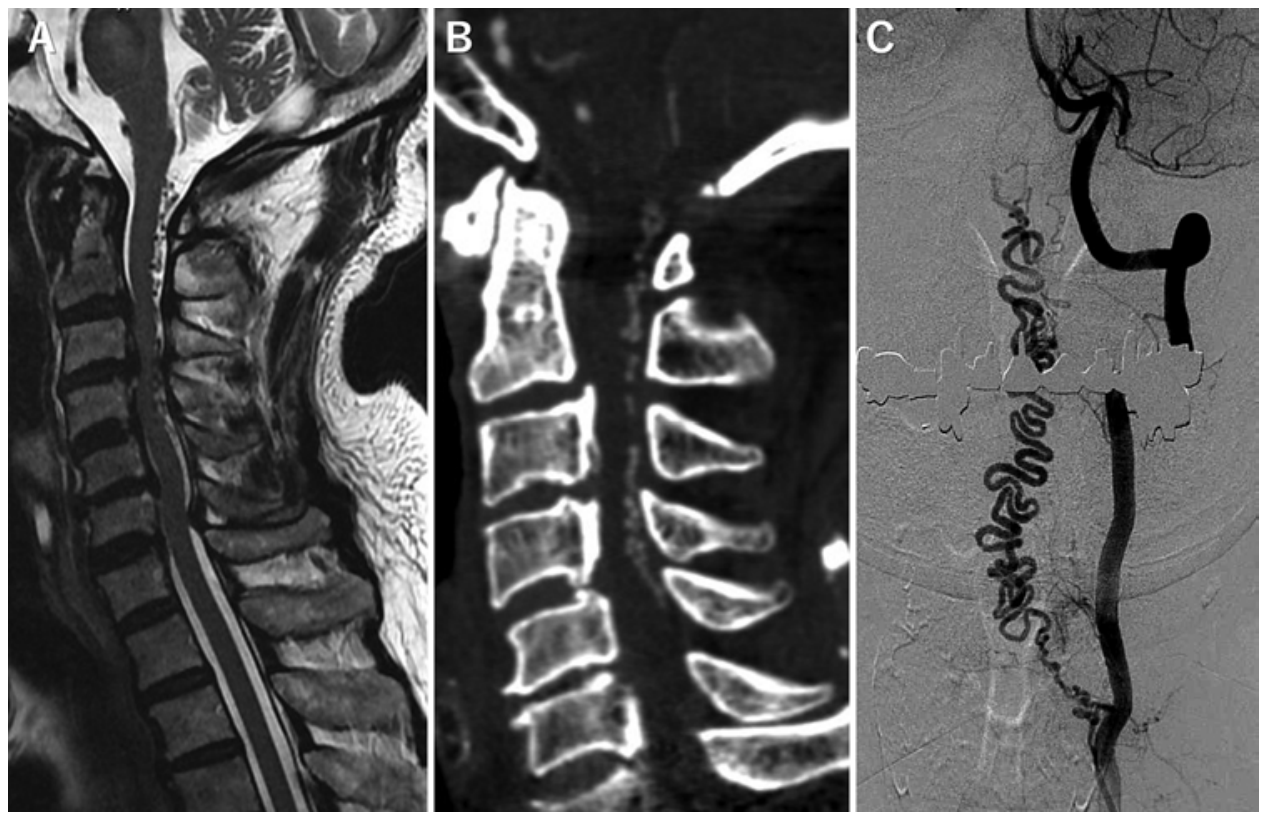

FIG. 2. A: Sagittal T2-weighted MR image of the cervical spine showing a serpentine and dilated perimedullary venous plexus on the dorsal spinal cord. B: Sagittal CT angiogram of the cervical spine showing engorgement of the perimedullary venous plexus and OPLL at the C3-4 levels. C: Left vertebral artery angiogram showing a dAVF fed by the left C6 segmental artery.

from the right T9 segmental artery (Fig. 4D). The feeding arteries from the branch of the ASA were embolized with $50 \%$ NBCA (n-butyl cyanoacrylate) with preservation of the ASA. An endovascular approach through the left T6 segmental artery was not possible because of the long and tortuous anatomy of the feeding artery. Postembolization angiography performed 1 month after the procedure showed no filling of the nidus from the right $\mathrm{T} 9$ segmental artery (Fig. 4E). Flow reduction was observed in the angiogram of the left T6 segmental artery (Fig. 4F).

We decided to perform surgery to occlude the residual supply from PSA of the left T6 segmental artery. Surgery was performed approximately 5 months after onset of paraplegia. The intraoperative selective angiography of the left T6 segmental artery showed intramedullary AVM (Fig. 4G). SAI-ICG showed the dilated feeding artery entering the spinal cord and the draining vein in the right side of the dorsal spine (Fig. 5A and B). Subsequently, SAIS and HFR recording were performed. The feeding artery was rendered transparent, and the red color of the proximal side of the draining vein was slightly diluted; however, the color of the distal side of the draining vein remained unchanged. The red color of the transparent feeding artery was restored by blood flow following the clearance of saline (Fig. 5C and D, Video 2).

VIDEO 2. High frame rate recording during SAIS shows the smooth and seamless motion of injected saline and blood returning to the vessels. Copyright Department of Neurosurgery, Hokkaido University Graduate School of Medicine. Published with permission. Click here to view.

A schematic illustration of the feeding artery and the draining vein is shown in Fig. 5E. Compared with SAIICG, HFR video provided better and more detailed information of the flow direction of the branch of the feeding artery. The direction of blood flow in the branch of the feeding artery (Fig. 5C, white arrowhead) was toward the cranial side (Fig. 5D, black arrowhead), which was hard to recognize with SAI-ICG. After the disconnection of the
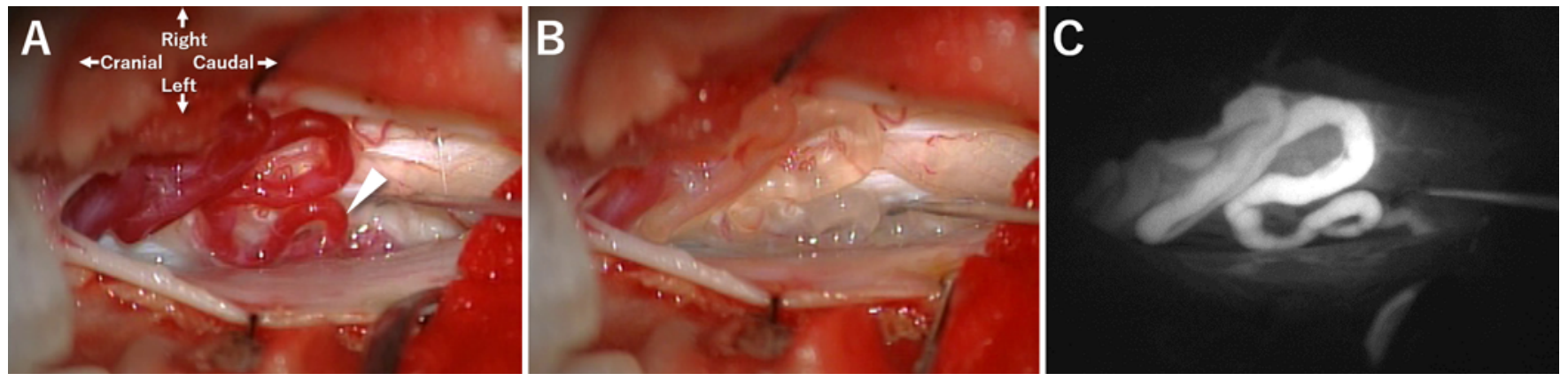

FIG. 3. A: Intraoperative photograph of the arterialized vein (white arrowhead) at the left C6 level. B: SAIS at the C6 segmental artery renders the vessel transparent. C: SAI-ICG at the C6 segmental artery allows visualization of the arterialized draining vein. 

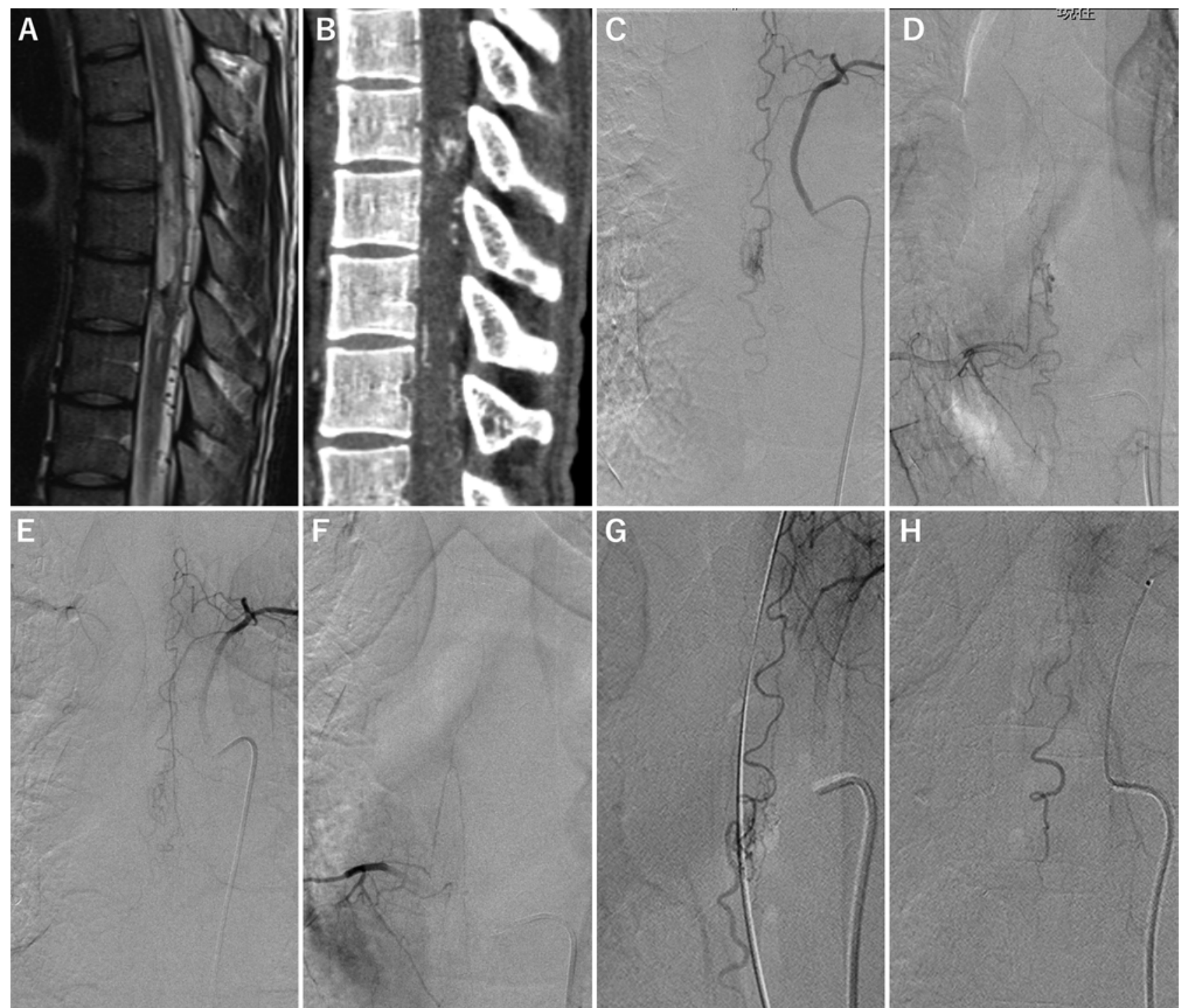

FIG. 4. A: Sagittal T2-weighted MR image shows posthemorrhagic change at T7-8 within the spinal cord. B: Sagittal CT angiogram shows a nidus of vessels at T7 within the spinal cord with an enlarged perimedullary venous plexus. C and D: Left T6 (C) and right T9 (D) segmental artery angiograms show filling of the nidus of vessels and early venous drainage. E: Postembolization angiogram of the left T6 segmental artery shows flow reduction to the nidus. F: Postembolization angiogram of the right T9 segmental artery shows no filling of the nidus. G: Intraoperative angiogram of the left T6 segmental artery shows an obvious filling of the nidus. H: Intraoperative angiogram of the left T6 segmental artery after the excision of the nidus confirms the disappearance of the nidus.

feeding artery at the entry point to the spinal cord, the nidus was resected for a radical cure. Postremoval angiography showed no evidence of residual AVM (Fig. 4H). The postoperative period was uneventful.

The patient's postoperative neurological function did not differ from preoperative levels, including complete paraplegia and bladder and rectal disturbances.

\section{Discussion}

Superselective arterial injection of ICG (SAI-ICG) is an effective method for the identification of feeding arteries and draining veins during surgery for spinal AVSs. ${ }^{2}$ The technique requires a very small amount of ICG, which is rapidly washed out, facilitating repeated examinations in a short period. Furthermore, SAI-ICG allows for visualization of the lesions from only the feeding artery, where the catheter is placed, while intravenous administration of ICG makes the whole lesion fluoresce at once. Due to these advantages, SAI-ICG is thought to be useful in the surgical treatment of complex AVS lesions such as intramedullary AVMs and perimedullary AVFs. Normally, the SAIS and HFR recording techniques are not necessary for the surgical treatment of dAVFs because of their simple angioarchitecture. Although we performed intraoperative angiography and SAIS in case 1 , intravenous administration of ICG was thought to be sufficient to elucidate the angioarchitecture of the lesion. We present this case of a dAVF to show that SAIS can render the abnormal vessels transparent in the bright field. 

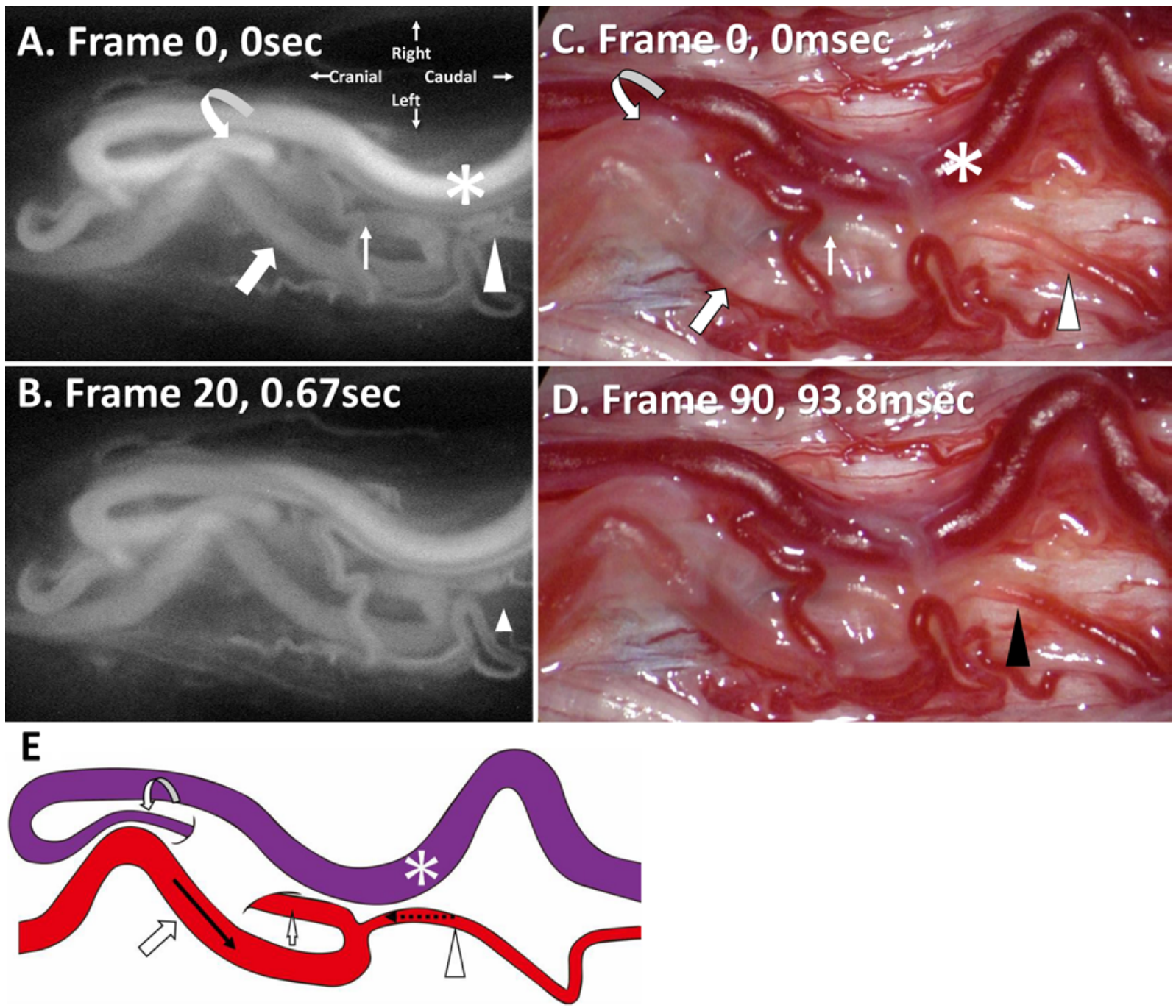

FIG. 5. A and B: SAI-ICG with a frame rate of 29.97 interlaced fps. Panel B indicates the captured video frame of 20 and 0.67 sec after panel A. Feeding artery (white arrow) and proximal and distal part of the draining vein (A, curved white arrow and asterisk, respectively) are identified. The fluorescent intensity of the branch of the feeding artery (A, white arrowhead) disappears between frames 0 and 20 (B, small white arrowhead). C and D: HFR recording with $960 \mathrm{fps}$ during SAIS. Panel D indicates the captured video frame of 90 and $93.8 \mathrm{msec}$ after panel $\mathrm{C}$. The feeding artery (C, white arrow) has become transparent with the injected saline. Blood returned into the feeding artery and the color of the feeding artery gradually becomes red (D). The red color of the proximal part of the draining vein (C, curved arrow) becomes thinner, whereas no obvious color change is observed in the distal part of the draining vein ( $\mathrm{C}$, asterisk). The injected saline is refluxed to the branch of the feeding artery ( $\mathrm{C}$, white arrowhead), which then flows back toward the main feeding artery (D, black arrowhead). E: Schematic illustration of the feeding artery and draining vein. Black and dotted black arrows indicate the flow direction of the feeding artery and the branch of the feeding artery, respectively.

One of the disadvantages of ICG angiography is the limited temporal resolution of recorded moving images. The recording frame rate of the operating microscope is usually set at $59.94 \mathrm{fps}$ in the bright field observing mode and at 29.97-59.94 fps in the NIR observing mode. When the frame rate is $59.94 \mathrm{fps}$, the frame-to-frame time is approximately $16.7 \mathrm{msec}$ and the temporal resolution is not sufficient for analyzing the blood flow. Allah et al. have reported a mean peak-systolic velocity of $28.1 \mathrm{~cm} / \mathrm{sec}$ in the ASA. ${ }^{1}$ According to the report, the estimated distance traversed by the blood in the spinal artery during the frameto-frame time is $28.1 \mathrm{~cm} / \mathrm{sec} \times 16.7 \mathrm{msec} / 1000=0.47 \mathrm{~cm}$, which is not a short distance in the microscopic field. Thus, recording with a higher frame rate allows a more detailed delineation of the blood flow. When the frame rate is 960 fps (frame-to-frame time, $1.04 \mathrm{msec}$ ), the time resolution is improved 16 times over that of a frame rate of $59.94 \mathrm{fps}$. In case 2 , the flow direction of the branch from the feeding artery was clearly determined in HFR recording during SAIS, which is hard to recognize during SAI-ICG; this indicates an advantage of HFR recoding over normal frame rate recording. In addition, our method is relatively easy and fast because the recorded video can be observed using ordinary movie player software after transferring the SD card from the camera to a personal computer.

There are some limitations of our method. The color 
change in vessels is not always observed in all branches of the vessel injected with saline because of the lower sensitivity of saline compared with that of ICG, as can be seen from case 2 . Therefore, the draining vein, which also serves as a normal drainage route, is typically hard to recognize during SAIS because the transparency conferred by saline is lost through intermixing with the normal venous flow. In addition, in the case of repeat surgery, the observation of vessel color change becomes difficult because of postsurgical adhesive tissue around the vessels. Therefore, the results of SAIS should always be compared with those of SAI-ICG. Finally, the true usefulness of the SAIS technique remains unclear due to the small number of cases in which it was used. It will be important to test the effectiveness of the technique in cases of spinal AVS with complex structures, such as many perimedullary AVFs.

\section{Conclusions}

We described SAIS and its usefulness and HFR recording during surgery for spinal AVSs. Our method has the following advantages: 1) The intraarterial injection of saline enables the real-time identification of the feeding artery within the bright field in combination with SAIICG. 2) Data recording with a digital camera allows quick and easy observation of the recorded video using ordinary movie player software on a personal computer. 3) HFR recording produces a smooth and seamless slow-motion video, thereby facilitating understanding of the blood flow.

\section{References}

1. Abd Allah F, Majidi S, Watanabe M, Chaudhry SA, Qureshi AI: Feasibility and validation of spinal cord vasculature im- aging using high resolution ultrasound. J Vasc Surg 56:637643,2012

2. Osanai T, Hida K, Asano T, Seki T, Sasamori T, Houkin K: Ten-year retrospective study on the management of spinal arteriovenous lesions: efficacy of a combination of intraoperative digital subtraction angiography and intraarterial dye injection. World Neurosurg 104:841-847, 2017

3. Simal-Julián JA, Miranda-Lloret P, Evangelista-Zamora R, Sanromán-Álvarez P, Pérez de San Román L, Pérez-Borredá $\mathrm{P}$, et al: Indocyanine green videoangiography methodological variations: review. Neurosurg Rev 38:49-57, 2015

\section{Disclosures}

The authors report no conflict of interest concerning the materials or methods used in this study or the findings specified in this paper.

\section{Author Contributions}

Conception and design: Hamauchi, Osanai. Acquisition of data: Hamauchi, Okamoto, Hida. Drafting the article: Hamauchi. Critically revising the article: Osanai. Administrative/technical/material support: Seki, Kawabori. Study supervision: Houkin.

\section{Supplemental Information \\ Videos \\ Video 1. https://vimeo.com/267394786. \\ Video 2. https://vimeo.com/267394803.}

\section{Correspondence}

Shuji Hamauchi: Hokkaido University Graduate School of Medicine, Sapporo, Japan. hamashuji@yahoo.co.jp. 